\title{
The Activity of the Postural Control System and Its Compensation in the Morbid Obese Subjects
}

\author{
M. BoseK ${ }^{a, *}$, S. Pierściński ${ }^{b}$, S. DAzBrowiecki $^{b}$ And B. Grzegorzewski ${ }^{a}$ \\ ${ }^{a}$ Department of Biophysics, Collegium Medicum in Bydgoszcz, Nicholaus Copernicus University \\ Jagiellońska 13, 85-067 Bydgoszcz, Poland \\ ${ }^{b}$ Department of General and Endocrine Surgery, University Hospital, Collegium Medicum in Bydgoszcz \\ Nicholaus Copernicus University, M. Skłodowskiej-Curie 9, 85-094 Bydgoszcz, Poland
}

The method based on two-dimensional stochastic process was used to examine the short-term postural sway under eyes-open and eyes-closed conditions. Obtained parameters, i.e., matrix of the fluctuation strength and friction coefficient, describe the stochastic activity of the muscles of the lower limbs as well as mechanisms compensating that activity. The standard force platform was used to measure the center of pressure displacements. In this investigation 17 control subjects and 22 morbid obese subjects were included. It is shown that the method is useful in the study of the postural control system of morbid obese subjects. The muscles responsible for postural control in these subjects have exhibited larger stochastic activity, than these muscles in non-obese subjects. However morbid obese as well as non-obese subjects compensate additionally enlarged level of muscles activity after closure of their eyes. The morbid obesity causes an increase of the muscles' force but does not cause the impairment of the postural control system.

PACS: 87.85.gj

\section{Introduction}

Many methods were proposed to analyze the postural sway under quiet-standing conditions [1-8]. One of them is the stabilogram-diffusion analysis, introduced by Collins and De Luca [9-13]. These authors have characterized the postural sway using the mean square displacement of the center of pressure (COP) as a function of time interval. They have found that, three different scaling regions, referred to as the short-term, long-term and saturation region, can be distinguished in the plot of this function. The scaling behavior in these regions suggest that the postural control system uses two different schemes: the open-loop control scheme over short-term intervals and the closed-loop control scheme over long-term intervals. Using this technique the stochastic activity of postural control system can be characterized. Collins and De Luca [11, 12] have found that activity over short-term intervals is the result of the stochastic activity of the muscles of the lower limbs. These findings were obtained for postural sway in anteroposterior (AP) as well as in mediolateral (ML) direction. It should be mentioned that observed scaling phenomenon has inspired many authors to use various advanced techniques to describe the COP sway [14-16].

Recently, as a model of the postural sway under quiet-standing conditions, the two-dimensional stochastic pro-

* corresponding author; e-mail: mbosek@cm.umk.pl cess was used $[17,18]$. This model describes the mean square COP displacement in the short-term region, thus it characterizes the open-loop postural control mechanism. As a result it has allowed the analysis of the activity of the short-term postural sway and in consequence the muscles' activity as well as the compensation of that activity. Furthermore, this activity was investigated in AP and ML directions, allowing the analysis of its orientation on the plane of support. This method was used to examine the postural control system for different populations under eyes-open and eyes-closed conditions $[17,18]$. It was shown that the muscular activity is increased, after exclusion of the visual input. On the other hand, this activity in elderly subjects is greater than that in young subjects. In the healthy elderly subjects, additionally increased activity under eyes-closed conditions, calls into play a compensating mechanism, whereas it is not observed in parkinsonian elderly subjects.

Balance of the obese subjects was already discussed. It was reported that obese and morbid obese subjects exhibit poorer postural stability [19-22]. Under quiet-standing conditions, it was observed as increased postural sway $[19,20]$. Postural instability in obesity is also observed as increased contribution of dual stance in gait cycle [20]. Teasdale et al. have shown that postural sway of obese and morbid obese subjects decreases and their stability improves with loss of their weight. Furthermore, McGraw et al. have found that postural control system of obese subjects is not impaired. It was frequently suggested that postural instability in obese subjects is due 
to increased mass of their body [19-21]. Additionally, in elderly subjects obesity accentuates effect of decreased muscles quality [22]. In case of obesity the effect of vision was also considered. Teasdale et al. have found that exclusion of vision causes similar changes in postural sway of non-obese, obese and morbid obese adults. However, McGraw et al. have reported that difference between postural sway of obese and non-obese prepubertal boys was accentuated without vision.

The aim of the present study was to examine the effect of the morbid obesity on the postural control system during quiet standing. Using the method based on the model of the stochastic process, we wanted to determine whether this system is impaired in morbid obese subjects.

\section{Materials and methods}

To describe the short-term dynamics of COP in ML and AP directions we have used two-dimensional Langevin equation of motion [23, 24], in the form

$$
\frac{\mathrm{d}^{2}}{\mathrm{~d} t^{2}} \boldsymbol{r}=-\gamma \frac{\mathrm{d}}{\mathrm{d} t} \boldsymbol{r}+\frac{1}{\sqrt{2}} \boldsymbol{G}^{1 / 2} \boldsymbol{f}(t),
$$

where $\boldsymbol{G}$ is the matrix of the fluctuation strength of the stochastic force, $\gamma$ is the friction coefficient and $\boldsymbol{f}(t)$ is the stochastic noise. This method is described in detail in previous investigations $[17,18]$. This model introduces as a parameter describing the postural sway, the diffusion matrix $\boldsymbol{D}=\boldsymbol{G} / 2 \gamma^{2}$, whose elements are roughly comparable with the short-term diffusion coefficient used by Collins and De Luca [9-13]. However, according to our method, this matrix can be separated into the direct measure of the stochastic activity of the short-term postural sway given by matrix $\boldsymbol{G}$ and friction coefficient $\gamma$ describing compensation of that activity. In this study the parameters characterizing the orientation of this activity were not presented. We have restricted our analysis only to the planar fluctuation strength of the stochastic force $\boldsymbol{G}_{r}=\operatorname{Tr} \boldsymbol{G}$, the planar diffusion coefficient $D_{r}=\operatorname{Tr} \boldsymbol{D}$ and the friction coefficient $\gamma$, where $\operatorname{Tr}$ is a trace of matrix.

In this study 17 non-obese subjects (4 male and 13 female) and 22 morbid obese subjects ( 7 male and 15 female) were included. In non-obese and morbid obese group, subjects had the following age: $41 \pm 8$ (31-59) and $42 \pm 7(32-56)$ years and body parameters: $68 \pm 16$ and $132 \pm 27 \mathrm{~kg}$ weight, $169 \pm 9$ and $170 \pm 10 \mathrm{~cm}$ height and $24 \pm 4$ and $46 \pm 9 \mathrm{~kg} / \mathrm{m}^{2}$ BMI, respectively, where only weight and BMI were significantly different. Investigated subjects have no history or evidence of any neurological disorders affecting movements control or posture. Morbid obese subjects were qualified to surgical treatment due to failure of medical treatment. All subjects were investigated at the same experimental conditions. Before tests, the subjects were asked to rest for some time to exclude the influence of fatigue. During test subject stood barefoot in an upright posture on the force platform, in a standardized stance with arms relaxed comfortably along the body. The feet of investigated subject were positioned along lines marked on the platform, heels together with an angle between the feet of $30^{\circ}$. The standard force platform, produced by Pro-Med (Janusz Olton, Poland), was used to measure the COP displacements at a rate of $32 \mathrm{~Hz}$. For each subject one test was performed with the eyes open and fixed on a wall in front of them, and one, with the eyes closed, and their order was randomized. Each test lasted for $32 \mathrm{~s}$ and the resting time between tests was about $30 \mathrm{~s}$. The experiment was undertaken according to the ethical guidelines laid down by the Bioethical Commission at the Ludwik Rydygier Collegium Medicum in Bydgoszcz of Nicolaus Copernicus University in Torun. Investigated subjects gave informed consent to the work. The theoretical functions were approximated to the experimental ones by least squares. T-test, for paired and for unpaired normal variables and Wilcoxon test, for paired, and Mann-Whitney test, for unpaired non-normal variables were used to compare parameters obtained for investigated groups in both experimental conditions. Normality of variables was tested using the Shapiro-Wilk test.

\section{Results}

To obtain the described above parameters for investigated subjects, we have fitted the matrix of the mean square displacement for theoretical model to that function for experimental data. The planar mean square displacement as a function of time interval for the representative morbid obese subject under eyes-open conditions and corresponding theoretical one is shown in Fig. 1. It is seen that, in the short-term region, i.e., for time intervals less than $1 \mathrm{~s}$, theoretical function approximates well function extracted from the COP time series.

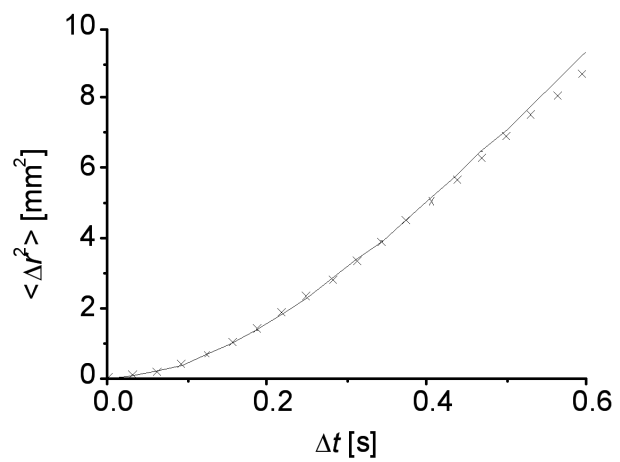

Fig. 1. The planar mean squared displacement $\left\langle\Delta r^{2}\right\rangle$ as a function of time interval $\Delta t$ obtained for representative morbid obese subject under eyes-open conditions (scatter plot) and, approximated to it, theoretical function (solid line).

The parameters describing the magnitude of the activity of the postural control system as well as the magnitude of the compensation of this activity, i.e., the planar diffusion coefficients $D_{r}$, the planar fluctuation strength $G_{r}$ and the friction coefficient $\gamma$, for both investigated 


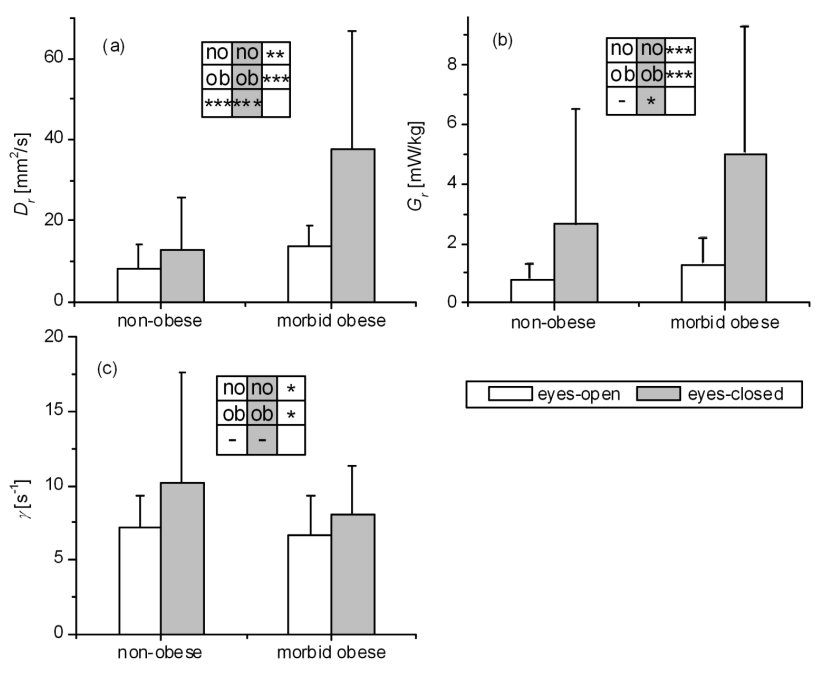

Fig. 2. The group means and standard deviations of the planar diffusion coefficient $D_{r}(\mathrm{a})$, the coefficient of the planar fluctuation strength of the stochastic force $G_{r}$ (b), the friction coefficient $\gamma$ (c) for non-obese (no) and morbid obese (ob) groups under eyes-open and eyes-closed conditions. In the tables on the plots, the significance levels of differences between groups and between visual conditions were shown at the end of the columns and rows, respectively, where the significance level at $P<0.001\left({ }^{* *}\right), P<0.01(* *)$ and $P<0.05\left(^{*}\right)$ or absence of significance $(-)$ were taken under consideration.

groups are shown in a bar-plot form in Fig. 2. Investigations are conducted under two visual conditions. The differences between non-obese and morbid obese group were examined through direct comparison and comparison of reaction on excluding of the visual input.

The diffusion coefficients $D_{r}$ for the morbid obese group were significantly greater than that for the non-obese group, under eyes-open as well as eyes-closed conditions $(P<0.001)$ (Fig. 2a). On the other hand, that coefficient for non-obese $(P<0.01)$ and morbid obese $(P<0.001)$ groups under eyes-closed conditions was significantly greater than that under eyes-open conditions. The group mean value of the planar diffusion coefficient for morbid obese subjects under eyes-open conditions and for non-obese subjects under eyes-closed conditions were approximately twice that for non-obese subjects under eyes-open conditions and were three times smaller than that for morbid obese subjects under eyes-closed conditions.

The planar fluctuation strength $G_{r}$ was significantly greater for morbid obese subjects than that for non-obese subjects under eyes-closed conditions $(P<0.05)$ (Fig. 2b). That difference is also observed under eyes-open conditions, however, it is not significant. For both groups $G_{r}$ significantly grows after closure of the subject's eyes $(P<0.001)$. The group mean value for both groups under eyes-closed conditions were even four time greater than that under eyes-open conditions.
For the friction coefficient $\gamma$ we have obtained similar result for both investigated groups. There were no significant differences for this coefficient between these groups (Fig. 2c). The friction coefficient for both groups under eyes-closed conditions was significantly greater than that under eyes-open conditions $(P<0.05)$. This increase indicates that postural control system uses mechanisms compensating increased muscles' activity under eyes-closed conditions for non-obese as well as morbid obese groups.

\section{Discussion and conclusions}

Using the method based on the model of the stochastic process, we have found that muscles responsible for postural control under quiet-standing conditions in morbid obese subjects have exhibited larger stochastic activity than these muscles in non-obese subjects. Furthermore, it was shown that morbid obese as well as non-obese subjects compensate additionally enlarged level of muscles activity after closure of their eyes. Consequently, morbid obesity causes an increase of the muscles' force but does not cause the impairment of the postural control system.

Observed increase of the activity of muscles responsible for postural control, indicating an increase of the force of these muscles, for morbid obese subjects, is probably caused by necessity of keeping in upright stance the body having greater mass. Similarly to our findings, Teasdale et al. [19] also have found increased postural sway in obese subjects. That increase was even greater in morbid obese subjects. However, obese and morbid obese subjects with loss of their weight diminished their postural sway to nearly normal level. One of the explanations of that phenomenon suggests that increased postural sway is due to increased muscular torque at ankle joints stabilizing the body with enlarged mass in abdominal area. This hypothesis is roughly consistent with ours which adopt assumption that, due to increased body mass, muscles, responsible for postural control, produce greater force. McGraw et al. [20] have obtained similar results for prepubertal boys. They have also shown that adiposity, due to increased weight, causes an increase of postural sway.

In both investigated groups after closing of the subject eyes the postural control system increased muscles' activity as well as used mechanism compensating this increase. This mechanism, appearing in healthy elderly subjects, causes the compensation of increased muscles' activity, when the increase due to age was superimposed on increase due to the exclusion of the visual input [18]. The absence of such mechanism in parkinsonian subjects of similar age to elderly group was a consequence of degeneracy of nervous system of these subjects. In this study we have shown that compensation mechanism appears in morbid obese and non-obese middle-aged subjects. It suggests that morbid obesity does not affect the postural control system. This is consistent with results of McGraw et al. [20], which have not found alterations in postural control system of obese prepubertal 
boys. Whereas, the increase of postural sway caused by the exclusion of the visual input, for morbid obese as well as for non-obese subjects, was also found in investigation of Teasdale et al. [19].

Finally, these investigations show validity of our method, also in case of morbid obesity. In this method the short-term postural sway under quiet-standing conditions is modeled, using the two-dimensional stochastic process. The advantage of this approach is good physiological interpretation of obtained parameters, which describe the stochastic activity of the muscles of the lower limbs as well as mechanisms compensating that activity.

\section{References}

[1] B. Grzegorzewski, A. Kowalczyk, Hum. Mov. Sci. 20, 853 (2001).

[2] S.S. Hasan, M.J. Lichtenstein, R.G. Shiavi, J. Biomech. 23, 783 (1990).

[3] M. Kuczyński, Gait Posture 9, 50 (1999).

[4] V.P. Panzer, S. Bandinelli, M. Hallet, Arch. Phys. Med. Rehabil. 76, 151 (1995).

[5] M.A. Riley, R. Balasubramaniam, M.T. Turvey, Gait Posture 9, 65 (1999).

[6] T. Shimba, J. Biomech. 17, 53 (1984).

[7] S. Thurner, Ch. Mittermaier, R. Hanel, K. Ehrenberger, Phys. Rev. E 62, 4018 (2000).

[8] D.A. Winter, F. Prince, J.S. Frank, C. Powell, K.F. Zabjek, J. Neurophysiol. 75, 2334 (1996).

[9] J.J. Collins, C.J. De Luca, Exp. Brain. Res. 95, 308 (1993).

[10] J.J. Collins, C.J. De Luca, Phys. Rev. Lett. 73, 764 (1994).
[11] J.J. Collins, C.J. De Luca, Exp. Brain Res. 103, 151 (1995).

[12] J.J. Collins, C.J. De Luca, A. Burrows, L.A. Lipsitz, Exp. Brain Res. 104, 480 (1995).

[13] S.L. Mitchell, J.J. Collins, C.J. De Luca, A. Burrows, L.A. Lipsitz, Neurosci. Lett. 197, 133 (1995).

[14] F. Alonso-Sanchez, D. Hochberg, Phys. Rev. E 62, 7008 (2000)

[15] M. Bosek, Biosystems 94, 282 (2008).

[16] T.D. Frank, A. Daffertshofer, P.J. Beek, Phys. Rev. E 63, 011905 (2000).

[17] M. Bosek, B. Grzegorzewski, A. Kowalczyk, Hum. Mov. Sci. 22, 649 (2004).

[18] M. Bosek, B. Grzegorzewski, A. Kowalczyk, I. Lubiński, Neurosci. Lett. 376, 215 (2005).

[19] N. Teasdale, O. Hue, J. Marcotte, F. Berrigan, M. Simoneau, J. Doré, P. Marceau, S. Marceau, A. Tremblay, Int. J. Obes. 31, 153 (2007).

[20] B. McGraw, B.A. McClenaghan, H.G. Williams, J. Dickerson, D.S. Ward, Arch. Phys. Med. Rehabil. 81, $484(2000)$

[21] K. Jadelis, M.E. Miller, W.H. Ettinger, S.P. Messier, J. Am. Geriatr. Soc. 49, 884 (2001).

[22] D.T. Villareal, M. Banks, C. Siener, D.R. Sinacore, S. Klein, Obes. Res. 12, 913 (2004).

[23] J.K.G. Dhont, An Introduction to Dynamics of Colloids, Elsevier, Amsterdam 1996.

[24] H. Risken, The Fokker-Planck Equation, Springer-Verlag, Berlin 1989. 